\title{
Energy Efficient Fault Tolerant Coverage in Wireless Sensor Networks
}

\author{
Shagufta Henna \\ Department of Computer Science, Bahria University, Islamabad, Pakistan \\ Correspondence should be addressed to Shagufta Henna; shinna.buic@bahria.edu.pk
}

Received 2 November 2016; Revised 18 February 2017; Accepted 19 February 2017; Published 3 April 2017

Academic Editor: Alberto J. Palma

Copyright (C) 2017 Shagufta Henna. This is an open access article distributed under the Creative Commons Attribution License, which permits unrestricted use, distribution, and reproduction in any medium, provided the original work is properly cited.

Energy efficiency and fault tolerance are two of the major concerns in wireless sensor networks (WSNs) for the target coverage. Design of target coverage algorithms for a large scale WSNs should incorporate both the energy efficiency and fault tolerance. In this paper, we study the coverage problem where the main objective is to construct two disjoint cover sets in randomly deployed WSNs based on relay energy $\left(E_{\text {relay }}\right)$. Further, we present an approximation algorithm called Energy Efficient Maximum Disjoint Coverage (EMDC) with provable approximation ratios. We analyze the performance of EMDC theoretically and also perform extensive simulations to demonstrate the effectiveness of EMDC in terms of fault tolerance and energy efficiency.

\section{Introduction}

WSN is a random or deterministic deployment of massive number of sensor nodes in a monitored area. WSNs have extensive applications, including military surveillance [1], target tracking, wild life monitoring, fire prevention, rescue operations, and air quality monitoring [2-4]. The primary goal of sensor nodes is to sense and collect raw data by monitoring particular environment, target, and barrier, and report that event to some sink node after some local processing or aggregation $[5,6]$.

Target coverage and network life time are two of the recent research trends in WSNs. Deployment of a set of sensors to cover a particular area, targets, or barrier is called coverage problem [7]. The main purpose of target coverage [8] is to continuously monitor a set of targets by using a subset of sensors. These sensor nodes are subject to failures due to various reasons. One of the common reasons is battery failure. Other reasons may include radio interference, software or hardware faults, and environmental changes. Sensor node failures may affect the coverage and connectivity adversely which in turn degrades the sensor network performance with higher network delays and higher energy consumption [9].

Sensor nodes are equipped with limited capacity batteries. Recently, it has been investigated whether it is possible to conserve energy by using duty cycle protocols, where nodes switch their radio on and off periodically prolonging the life time of a WSN. Due to one time battery life and difficulty of replacing it, sensor nodes are densely deployed to increase connectivity and target coverage. However, if all the nodes are constantly on, it may quickly deplete their battery and may affect the network life time. Therefore it is important to tune these sensors to duty-cycled mode where they alternate between the active and sleep periods. Further, sensor hardware or software may fail due to weather or other physical conditions in a WSN affecting coverage of target nodes. If coverage of the target nodes is achieved by a single set of covering nodes, they may soon deplete their energy affecting the network life time. Therefore, it is important for WSNs to use redundant or disjoint covering sensors to cover particular area, targets, or barriers to construct a fault tolerant network which may still cover the targets, area, or barrier despite the failure of some covering sensors.

In order to effectively utilize the sensor nodes, some studies have selected a number of cover sets to provide coverage to some target sets. These studies organize the cover sets $[7,10]$ into disjoint or nondisjoint cover sets $[11,12]$. The selection of such disjoint or nondisjoint cover sets is proved to be NP-complete problem $[9,11]$. Disjoint or nondisjoint cover sets monitor the targets in a cooperative way. Compared to nondisjoint cover sets, disjoint cover sets are better at fault 
tolerance because all the cover sets have exclusive sensor nodes among their sets. Each cover set can alternatively work to maximize the network lifetime.

A number of studies have addressed the issue of target coverage; however, only fewer of them considered the disjoint target coverage based on minimum relay energy. In this article, our key focus is on the fault tolerant target coverage problem with minimum relay energy. We formulate the disjoint target coverage problem based on $E_{\text {relay }}$ energy. This problem differs from the target coverage which is based on disjoint covering sets, since coverage ensures that the $E_{\text {relay }}$ energy of each sensor from each of the disjoint sets is kept at minimum. We aim to select two disjoint covering sets with minimum $E_{\text {relay }}$ to cover the targets; that is, each sensor has minimum $E_{\text {relay }}$ energy. It is crucial to select sensor nodes which consume minimum energy to report an event to the sink node. Therefore, our primary objective is to maximize the network lifetime by selecting two disjoint covering sets with minimum overall $E_{\text {relay }}$ energy. In order to fulfil both the fault tolerance and energy efficiency requirements, we propose an efficient approximation algorithm, Energy Efficient Maximum Disjoint Coverage (EMDC), with provable approximation bound. Our key contributions in this paper are summarized as follows:

(i) We present a comprehensive comparison of well known target coverage, area coverage, and barrier coverage schemes.

(ii) We formulate disjoint coverage problem with minimum $E_{\text {relay }}$ energy.

(iii) We propose an efficient bounded approximation algorithm, the EMDC, to the minimum $E_{\text {relay }}$ energy coverage problem and present an appropriate theoretical analysis of the EMDC approximation ratio.

(iv) We perform simulations under different scenarios to establish the effectiveness of EMDC.

The remaining article is organized as follows: Section 2 gives a comprehensive background of the coverage and presents a comparison of different coverage schemes in WSNs. Section 3 summarizes the related work followed by the formulation of minimum energy disjoint coverage problem in Section 4. Section 5 presents EMDC algorithm design. Section 6 presents the theoretical analysis of EMDC. Section 7 presents the EMDC simulation results, and, finally, Section 8 concludes the paper.

\section{Coverage in WSNs}

A sensor is a device with the capability of responding to different physical stimuli including sound, heat, smoke, pressure, and any other event and transforming it into corresponding electrical or mechanical signal [13]. These signals are mapped to sensor information. A sensor node consists of one or more sensing units, battery, memory, data processing unit, and data transmission unit. A WSN consists of different sensing nodes placed in an area to detect or monitor certain activity. One of the most recent trends in WSNs research is the coverage question which reflects how well a particular area, target, or barrier is monitored. Coverage problems in WSNs can arise during the network design, deployment, or operation [14]. During the design of the network, coverage questions can be addressed by deciding the number of sensors to cover a particular area. In WSN deployment, sensors are deployed to achieve the coverage of desired targets, barriers, or areas in a geographical region. During the operational phase of a sensor network, a schedule is decided to conserve energy and increase network life time. Sensor coverage problems can be divided into three categories:

(i) Area coverage: [15-18], where the primary objective is to continuously observe or cover some particular area or whole sensor field.

(ii) Target coverage: [19], where the key objective is to monitor some particular points also termed as targets.

(iii) Barrier coverage: [20], a barrier coverage is a circular area where the presence of any intruder can be detected by a set of sensor deployed in this area.

Table 1 summarizes different coverage approaches with type of coverage and objectives in WSNs. Our work in this paper focuses on targeting coverage only.

2.1. Target Coverage Problems. In target coverage, the primary objective is to cover some particular set of points or targets deployed in a sensor field, for example, missile launchers in a battlefield. These targets can be covered by using a random or deterministic placement of sensors.

Optimal Placement of Sensors. In the deterministic approach to node placement, nodes are placed at predetermined locations to cover targets. The deterministic approach to node placement is convenient to use for reachable and friendly sensor fields. The main objective of this approach is to cover optimal locations by using a minimum number of covering nodes. In this technique, it is assumed that the locations of targets to be monitored are fixed, known, and are limited. In some cases, coverage of all the targets is not necessary, when the number of covering sensors is limited or it is expensive to cover them. Most of the problems related to sensor placement are optimization problems, and it is possible to formulate them as mathematical programming problems. However, greedy solutions may not produce the best possible placement. The problem to construct minimum number of disjoint sensors to cover targets is a well-known set cover problem [37]. Covering sensors can be represented as set covers used to cover particular deployed targets or area. To place a covering sensor, it must be placed on a location to cover at least one target, and it is possible to cover all the targets if the covering sensors are deployed on all the available locations. Different variants of the greedy approach for set cover are well documented in literature to solve various problems related to optimal node placement [38-41]. Apart 
TABLE 1: Coverage approaches used in WSNs.

\begin{tabular}{|c|c|c|}
\hline Method & Type of coverage & Main objectives \\
\hline Disjoint dominating sets [21] & Area coverage & Maximize lifetime and energy of a WSN \\
\hline $\begin{array}{l}\text { Coverage configuration protocol (CCP) } \\
{[15]}\end{array}$ & Area coverage & Improve connectivity and energy efficiency \\
\hline Coverage based on CDS [22] & Area coverage & Enhance network lifetime and energy of a WSN \\
\hline Placement algorithm for nodes [19] & $\begin{array}{l}\text { Area coverage and } \\
\text { Target coverage }\end{array}$ & Coverage and connectivity \\
\hline Disjoint set cover algorithm [23] & Target coverage & Energy efficient strong coverage \\
\hline $\begin{array}{l}\text { Density control algorithm based on } \\
\text { probing [24] }\end{array}$ & Area coverage & Energy efficient strong coverage \\
\hline $\begin{array}{l}\text { Optimal geographical density control } \\
\text { (OGDC) algorithm [25] }\end{array}$ & Area coverage & Energy efficient strong coverage \\
\hline Self-scheduling algorithm for nodes [16] & Area coverage & Energy efficient strong coverage \\
\hline OSRCEA Algorithm [26] & Area coverage & Improved coverage and connectivity \\
\hline $\begin{array}{l}\text { Voronoi-based coverage improvement } \\
\text { approach [27] }\end{array}$ & Area coverage & Improved coverage ratio \\
\hline $\begin{array}{l}\text { Localized algorithm for hole detection } \\
\text { and healing [28] }\end{array}$ & Area coverage & Coverage hole detection and healing \\
\hline $\begin{array}{l}\text { Optimal angular coverage in video sensor } \\
\text { networks [29] }\end{array}$ & Area coverage & Improved angular area coverage \\
\hline $\begin{array}{l}\text { Approximation algorithm for surface } \\
\text { coverage [30] }\end{array}$ & Surface coverage & Improved surface coverage \\
\hline $\begin{array}{l}\text { Particle swarm optimization (PI-BPSO) } \\
\text { algorithm [31] }\end{array}$ & Camera coverage & $\begin{array}{l}\text { Improved homogeneous camera network } \\
\text { coverage }\end{array}$ \\
\hline $\begin{array}{l}\text { Delaunay-based coordinate-free } \\
\text { mechanism (DECM) [32] }\end{array}$ & Area coverage & Coverage hole detection and healing \\
\hline Full-view coverage detection [33] & Area coverage & Full view coverage detection \\
\hline Detection accuracy algorithm [34] & Target coverage & Achieve detection accuracy \\
\hline $\begin{array}{l}\text { Minimum weight barrier algorithm } \\
\text { (MWBA) [26] }\end{array}$ & Barrier coverage & Increase detection probability of barriers \\
\hline Strong barrier coverag algorithm [35] & Barrier coverage & $\begin{array}{l}\text { Strong barrier coverage with minimum } \\
\text { directional sensors }\end{array}$ \\
\hline $\begin{array}{l}\text { A greedy solution for } k \text {-barrier coverage } \\
{[36]}\end{array}$ & Barrier coverage & To construct minimum size $k$-barrier coverage \\
\hline
\end{tabular}

from greedy algorithms, several approximation solutions have also been proposed for node placement $[42,43]$.

Coverage Lifetime Maximization. In a random placement, sensors are randomly scattered to cover targets. In a random deployment, a single sensor node may cover multiple fixed targets, and a fixed target may be covered by multiple deployed sensors. Deployment of sensors in a random placement may be dense. The coverage lifetime maximization problem which is a distinct version of the target coverage problem is to partition the sensors into more than one set covers subject to certain coverage requirements and to activate these set covers alternately to increase the network lifetime. An example of random deployment of target coverage is illustrated in Figure 1(a), where 6 sensing devices are deployed to cover 4 targets in a random setting. In Figure 1(a) the $T_{2}$ and $T_{4}$ targets are covered by two sensors, and $T_{1}$ and $T_{3}$ are covered by three sensor nodes. The coverage relationship between the sensors and targets can be depicted by a bipartite graph as illustrated in Figure 1(b).
To achieve target coverage, all the sensors can be activated which is not very energy efficient and may reduce the network lifetime. However alternatively activating the sensors may prolong the network lifetime. Assume that if all the sensors are activated for one unit of time, it will consume one unit of network lifetime. In Figure 1, we have two disjoint set covers $S_{1}=\left\{s_{1}, s_{3}, s_{6}\right\}$ and $S_{2}=\left\{s_{2}, s_{4}, s_{5}\right\}$ to cover all the targets. For one time unit set $S_{1}$ can cover targets, and, for the other $S_{2}$, increase the network lifetime to two time units. Using an optimal number of set covers and alternatively activating them may increase the network lifetime.

Another version of the target coverage is Maximum Set Cover (MSC), in which the primary objective is to cover all the specified targets all the time. The MSC problem is known to be $\mathscr{N} \mathscr{P}$-complete [44]. Target coverage at all times is a strict requirement for the coverage. The $k$-set cover for minimum coverage breach problem allows coverage breach and relaxes the strict coverage requirement [45]. A breached target is not covered by any sensor, or in other words breach coverage requires partial target coverage only. In this 


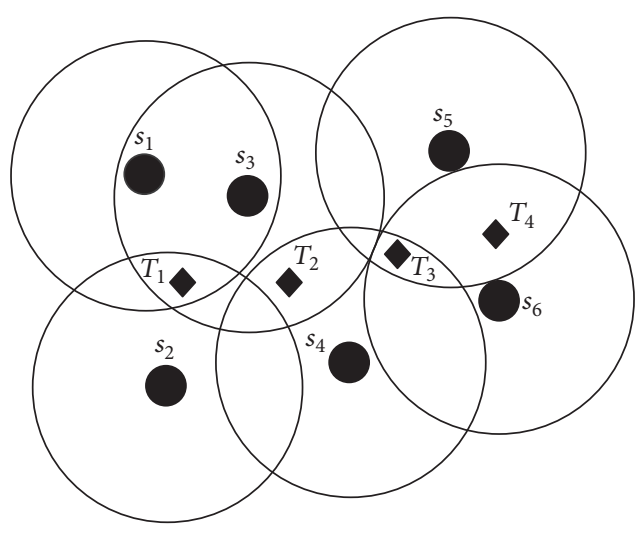

(a)

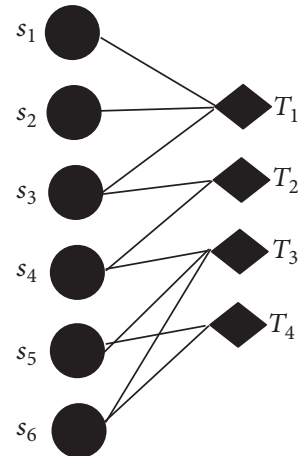

(b)

Figure 1: (a) Random deployment of sensor nodes. (b) Corresponding bipartite graph for (a).

problem, set covers are computed to cover only a fraction of fixed targets and are activated alternatively for short duration. The main objective of the $k$-set cover problem is to increase the lifetime of a WSN by constructing maximum $k$ set covers [45-47]. In [45], a problem called disjoint set covers (DSC) has been proposed for complete target coverage which is similar to the MSC problem with disjointness constraints. Energy efficiency using disjoint set covers to alternatively perform the coverage task has been discussed in $[18,44]$.

Slijepcevic and Potkonjak [18] proposed a centralized solution for the $k$-coverage problem, where points enclosed in a particular area called fields are monitored by $k$ set of sensors. The algorithm [18] covers the most critical fields by using maximum possible set covers. In this algorithm, the set covers which can cover a high number of uncovered points in an area are given priority. This algorithm also avoids field coverage redundancy. There exist several distributed solutions which can achieve 1-coverage, that is, can cover the target or area by using only one set cover. In [16], a pruning method is presented, where each node switches off its radio to conserve energy if its area can be covered by some of its neighbours. Unlike a centralized solution for the $k$-coverage problem in [18], a distributed solution for the same problem has been proposed in [46].

In our work, we formulate a decision version of the target coverage problem called EMDC. The EMDC problem is to achieve the maximum disjoint coverage using two disjoint set covers $D_{1}$ and $D_{2}$ with minimum $E_{\text {relay }}$ energy. Our problem aims to find a set cover $D_{2}$ with minimum $E_{\text {relay }}$ energy to maximize the target coverage in such a way that disjoint set cover $D_{1}$ with minimum $E_{\text {relay }}$ still completely covers all the targets. Further, $E_{\text {relay }}$ of $D_{1}$ is less than or equal to $E_{\text {relay }}$ energy of $D_{2}$.

\section{Related Works}

Recently, there is a trend in WSNs to discover set covers that are disjoint and are connected with a sink for target reporting. These disjoint sets can be activated alternatively to conserve energy or can be activated simultaneously to ensure reliable coverage. I. Cardei and M. Cardei [48] addressed the problem known as Connected Set Covers (CSC) problem which computes the maximum possible set covers connected to a sink node and proved this problem as an NP-complete problem. To address this problem, authors proposed two different heruistics based on integer programming and breadth-first search. Ostovari et al. presented an edge-cost based approach for the connected point coverage [49]. In this approach, a node can decide to act as a relay node by evaluating its edge cost in the Minimum Spanning Tree (MST) [50, 51].

Zhao and Gurusamy formulated the connected target coverage problem as maximum cover tree (MCT) problem [52]. They proved MCT problem as an NP-complete problem and established an upper bound for the network lifetime. Zorbas et al. [53] also considered the target coverage problem and proposed a power efficient coverage algorithm to select disjoint and nondisjoint cover sets with particular focus on weakly monitored targets. Yu et al. [54] presented a $k$-coverage working set construction (CWSC) algorithm. CWSC algorithm is able to produce different degrees of coverage according to the application selected. In [55], Shih et al. modelled the target coverage problem by considering multiple sensing units. They reduced it to a connected set cover problem. Further, they presented two distributed heuristics: the energy efficiency first scheme (EEFS) and remaining energy first scheme (REFS). In [56], authors have proposed a centralized algorithm called $\mathrm{CCTC}_{k}$ and a distributed algorithm called DCTC $k$ for connected target $k$ coverage problem in heterogeneous WSNs. Both algorithms construct connected cover sets to achieve connectivity and coverage in an energy efficient way.

Zhao and Gurusamy [52] proposed a distributed approximation algorithm known as Communication Weighted Greedy Cover (CWGC). The primary objective of the CWGC is to maximize the covering sets by considering minimum total weight from each node to the sink node. CWGC algorithm maximizes network lifetime compared to some existing work; however it does not take into account poorly covered targets. Yang et al. considered the special instance of $k$-connected coverage and designed two distributed solutions 
based on clustering and pruning [57]. In [58], authors have considered the target coverage problem by considering two types of sensor nodes including resource rich sensors and energy-constrained sensors.

Zorbas and Razafindralambo [34] proposed Optimized Connected Coverage Heuristic (OCCH) to compute set covers, and each node is assigned a weight which exempts the critical nodes from acting as relay nodes. OCCH prolongs network lifetime by conserving the energy of energy-constrained node. Experiments reveal that $\mathrm{OCCH}$ has better lifetime compared to CWGC. Some other popular approaches which satisfy the energy and connectivity have been discussed in $[34,59]$.

Henna and Erlebach [60] formulate a problem called Maximum Disjoint Coverage Problem (MDC). Main objective of MDC is to construct two disjoint set covers $S_{1}$ and $S_{2}$, where $S_{1}$ completely covers all the targets and $S_{2}$ covers the maximum of these targets. Authors proved the problem as an NP-complete problem and presented an efficient approximation algorithm called DSC-MDC to solve it. However, DSC-MDC just computes the disjoint set covers but does not consider the relay energy of the sensor nodes. DSC-MDC therefore is not an energy efficient fault tolerance solution to target coverage.

Coverage, connectivity, and fault tolerance are primary objectives for most of the target tracking sensor networks. However, in order to achieve connectivity and coverage, we cannot ignore critical nodes, especially nodes with critical energy. Hence, it is necessary to select disjoint set covers which take into account $E_{\text {relay }}$ energy of nodes to report an event to a particular sink. However, as discussed in the aforementioned work, most of the existing schemes discussing target coverage problem to compute cover sets ignore the issue of critical nodes as relay nodes. In this study, we propose an approximation Energy Efficient Maximum Disjoint Coverage (EMDC) algorithm to construct two disjoint cover sets to achieve both the fault tolerance and energy efficiency. We establish that approximation ratio of EMDC is $\sqrt{m}$, where $m$ denotes the number of fixed targets.

\section{Preliminaries}

4.1. Set Cover Problem. Let $U$ denote the set of elements and $C$ be a collection of subsets of set $U$. Given both $U$ and $C$, the set cover problem targets to cover all the elements of set $U$ by selecting the minimum number of sets from $C$. Basically, set cover problem aims to cover all the elements of set $U$. The set cover problem is illustrated below with the help of an example.

Let $U=\{a, b, c, d\}$ be the universal set. Given the following collection of sets $S, S=\left\{S_{1}, S_{2}, S_{3}, S_{4}\right\}$, where $S_{1}=$ $\{a, b\}, S_{2}=\{b, c\}, S_{3}=\{c, d\}$, and $S_{4}=\{d, a\}$. Both $S_{1}$ and $S_{3}$ together form a minimum set cover, that is, Set_cover ${ }_{1}=$ $\left\{S_{1}, S_{3}\right\}$. Another possible minimum set cover is Set_cover $_{2}=$ $\left\{S_{2}, S_{4}\right\}$. Both minimum set covers have size 2 .

4.2. Disjoint Set Covers (DSC). Given a finite set of sensor nodes $W$ to cover a finite set of targets, $T$, the DSC problem is to construct disjoint set covers from $W$ to cover all the elements of $T$ [23]. A set cover $W_{i} \subset W$ is selected such that $t_{j} \in T$ is covered by at least one of the elements of $W_{i}$ and for $W_{i}$ and $W_{k}, W_{i} \cap W_{k}=\emptyset$.

4.3. Maximum Disjoint Coverage Problem (MDC). Given a set $W$ of subsets of a given finite $\operatorname{target}$ set $T$, construct two disjoint set covers $W_{1}$ and $W_{2}$ for $T$, such that each element of target $T$ is covered by at least one element of set $W_{1}$, and an element of $W_{2}$ covers the maximum elements of $T$, and, for the set covers $W_{1}$ and $W_{2}, W_{1} \cap W_{2}=\emptyset$.

The decision variation of the MDC called Disjoint Coverage (DC) is defined as follows.

Disjoint Coverage (DC). Given a finite set of targets $T$ for a collection $W$ of subsets of $T$, determine if $S$ can be divided into two set covers with no common element, and these set covers can completely cover all the elements of set $T$.

4.4. Energy Efficient Disjoint Target Coverage Problem $(E M D C)$. Given a collection of $W$ of subsets with $E_{\text {relay }}$ energy of a given set $T$, EMDC problem seeks two disjoint cover set $D_{1}$ and $D_{2}$ for $T$ such that the following objectives are achieved:

(i) Each element of $T$ is being covered by at least one element of $D_{1}$.

(ii) $D_{2}$ covers maximum elements of $T$.

(iii) Set covers $D_{1}$ and $D_{2}, D_{1} \cap D_{2}=\emptyset$.

(iv) $E_{\text {relay }}$ energy of $D_{1}$ is less than or equal to $E_{\text {relay }}$ energy of $D_{2}$.

\section{Energy Efficient Maximum Disjoint Coverage (EMDC)}

EMDC is an approach to the fault tolerant energy efficient problem and is performed by each sensor. EMDC selects two energy efficient disjoint sets $D_{1}$ and $D_{2}$ based on the following principles: (i) select a sensor node as a candidate for $D_{1}$ or $D_{2}$ if it can cover maximum possible targets; (ii) favour sensor nodes that consumes minimum energy to report an event to the sink node; (iii) make sure both the set covers $D_{1}$ and $D_{2}$ cover the target nodes $T$ and are completely disjoint, with no common nodes.

It is worth mentioning that EMDC favours a sensor node to be added in the set cover $D_{1}$ or $D_{2}$ depending on the $E_{\text {relay }}$ energy which is calculated according to (1). For a subset $M_{i}$ representing neighbours of node $i, E_{\text {Relay }_{i}}\left(M_{i}\right)$ is calculated according to

$$
E_{\text {relay }_{i}}\left(M_{i}\right)=\frac{1}{\left|M_{i}\right|}+\frac{\sum_{j \in M_{i}} E_{\text {relay }_{j}}}{\left|M_{i}\right|} .
$$

In (1), the first term denotes the expected waiting time a node from a set of sensor nodes $W$ takes to report an event to a fixed sink node and is inversely proportional the number of its 1-hop neighbours. The second terms represents the average 
expected time a node has to wait to report the event to a particular sink node $B . E_{\text {relay }_{i}}\left(M_{i}\right)$, therefore, represents the total expected waiting time a node will take to report the event to the sink node $B . E_{\text {relay }_{i}}\left(M_{i}\right)$ is a better choice to select the nodes with less energy consumption to report an event to a particular sink node.

EMDC determines two energy efficient disjoint set covers $D_{1}$ and $D_{2}$ with minimum $E_{\text {relay }}$ energy. Algorithm first constructs $D_{2}$ by including sensor nodes which have minimum $E_{\text {relay }}$ energy and can cover the maximum elements of set $T$. On the other hand, $D_{1}$ includes sensor nodes with minimum $E_{\text {relay }}$ energy and are able to cover all the given targets from the set $T$. In order to compute two disjoint sets $D_{1}$ and $D_{2}$, algorithm takes subsets $T=\left\{t_{1}, t_{2}, \ldots, t_{m}\right\}$ and $W=$ $\left\{w_{1}, w_{2}, \ldots, w_{n}\right\}$. EMDC assumes that each subset from the collection $W$ knows its $E_{\text {relay }}$ energy. Each subset from the collection $W$ is mapped to a certain number of subsets from the collection $T$. EMDC selects a set $w_{i}$ from the collection $W$ by using a greedy approach. EMDC selects $w_{i}$ from $W$ to be added into the set $D_{2}$ if $w_{i}$ covers the maximum number of elements from the set $T$ and has minimum $E_{\text {relay }}$ energy, and there exists a set $w_{j}$ which covers the same or more number of targets as $w_{i}$. Further, EMDC evaluates if $w_{j}$ has less or equal $E_{\text {relay }}$ energy as $w_{i}$, if it is true, EMDC adds the set $w_{i}$ in $D_{2}$. The algorithm EMDC repeats until all the possible subsets from the collection $W$ have been added in the set cover $D_{2}$. Subsets in $W$, that is, $W \backslash D_{2}$, are passed to the $\operatorname{DisjointSet}(W)$ algorithm. DisjointSet $(W)$ adds a subset $w_{i}$ in set $D_{1}$ if it covers maximum targets from the set $T$ and has minimum $E_{\text {relay }}$ energy among all the subsets covering the same number of targets. Dis jointSet $(W)$ repeats until all the targets are covered from $T$. At the end, DisjointSet $(W)$ returns a disjoint set cover $D_{1}$ with minimum $E_{\text {relay }}$ energy which covers all the targets from the set $T$. Finally, EMDC returns $D_{1}$ and $D_{2}$ set covers with minimum $E_{\text {relay }}$ energy, where $D_{1}$ covers all the targets from the set $T$, and $D_{2}$ covers maximum of them. Algorithm EMDC and its counterpart DisjointSet $(W)$ are shown in Algorithms 1 and 2.

We can explain the operation of Algorithm 1 with an example given in Figure 2. Figure 2 shows a bipartite graph with two sets $W$ and T. $W$ is a covering set which can cover the elements in set $T$. The coverage relationship between the elements of set $W$ and $T$ is illustrated with the help of an edge. From the bipartite graph given in Figure 2, Algorithms 1 and 2 compute two disjoint set covers $D_{1}$ and $D_{2}$. follows:

Algorithm 1 computes $D_{2}$ to cover the set of targets $T$ as

(1) EMDC chooses $w_{2}$ greedily to cover maximum targets, that is, $\left\{t_{1}, t_{2}, t_{3}, t_{4}\right\}$, such that still it is possible to cover these targets by $w_{3}$ and $w_{5}$ which has less $E_{\text {relay }}$ than $w_{2}$ and adds $w_{2}$ to $D_{2}$.

$$
D_{2}=\left\{w_{2}\right\}
$$

(2) EMDC chooses $w_{6}$ greedily to cover maximum targets, that is, $\left\{t_{4}, t_{5}, t_{6}\right\}$, such that still it is possible to

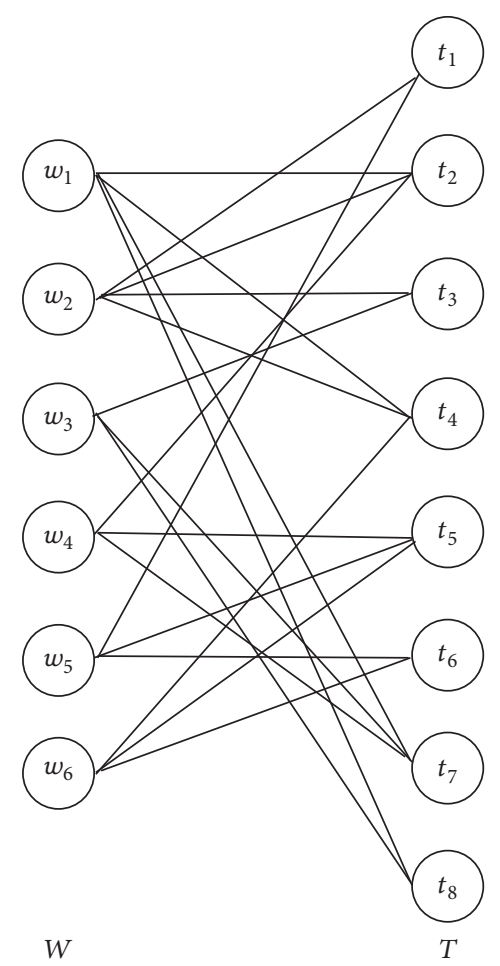

FIGURE 2: A bipartite graph with two energy efficient disjoint set covers.

cover these targets by $w_{1}$ and $w_{5}$ having less $E_{\text {relay }}$ energy compared to $w_{6}$ and adds $w_{6}$ to $D_{2}$.

$$
D_{2}=\left\{w_{2}, w_{6}\right\} \text {. }
$$

(3) EMDC chooses $w_{4}$ greedily to cover maximum targets, that is, $\left\{t_{2}, t_{5}, t_{7}\right\}$, such that still it is possible to cover these targets by $w_{1}$ and $w_{5}$ which has less $E_{\text {relay }}$ energy compared to $w_{4}$ and adds $w_{4}$ to $D_{2}$. EMDC does not select $w_{3}$ or $w_{1}$ because then $t_{3}$ or $t_{4}$ cannot be covered by $D_{1}$.

$$
D_{2}=\left\{w_{2}, w_{6}, w_{4}\right\}
$$

Targets covered by set cover $D_{2}$ are

$$
\begin{aligned}
T & =\left\{t_{1}, t_{2}, t_{3}, t_{4}\right\} \cup\left\{t_{4}, t_{5}, t_{6}\right\} \cup\left\{t_{2}, t_{5}, t_{7}\right\} \\
& =\left\{t_{1}, t_{2}, t_{3}, t_{4}, t_{5}, t_{6}, t_{7}\right\} .
\end{aligned}
$$

From the remaining sets, that is, $\left\{w_{1}, w_{2}, w_{3}\right\}$, Algorithm 2 computes $D_{1}$ greedily to cover the set of targets $T$ as follows:

$$
D_{1}=\left\{w_{1}, w_{5}, w_{3}\right\}
$$

Targets covered by set cover $D_{1}$

$$
\begin{aligned}
T & =\left\{t_{2}, t_{4}, t_{7}, t_{8}\right\} \cup\left\{t_{1}, t_{5}, t_{6}\right\} \cup\left\{t_{3}, t_{7}, t_{8}\right\} \\
& =\left\{t_{1}, t_{2}, t_{3}, t_{4}, t_{5}, t_{6}, t_{7}, t_{8}\right\} .
\end{aligned}
$$

In this example, $D_{1}$ and $D_{2}$ are selected disjoint set covers. By using Algorithms 1 and 2, $D_{1}$ achieves complete coverage of set $T$, and $D_{2}$ covers maximum elements of set $T$. 


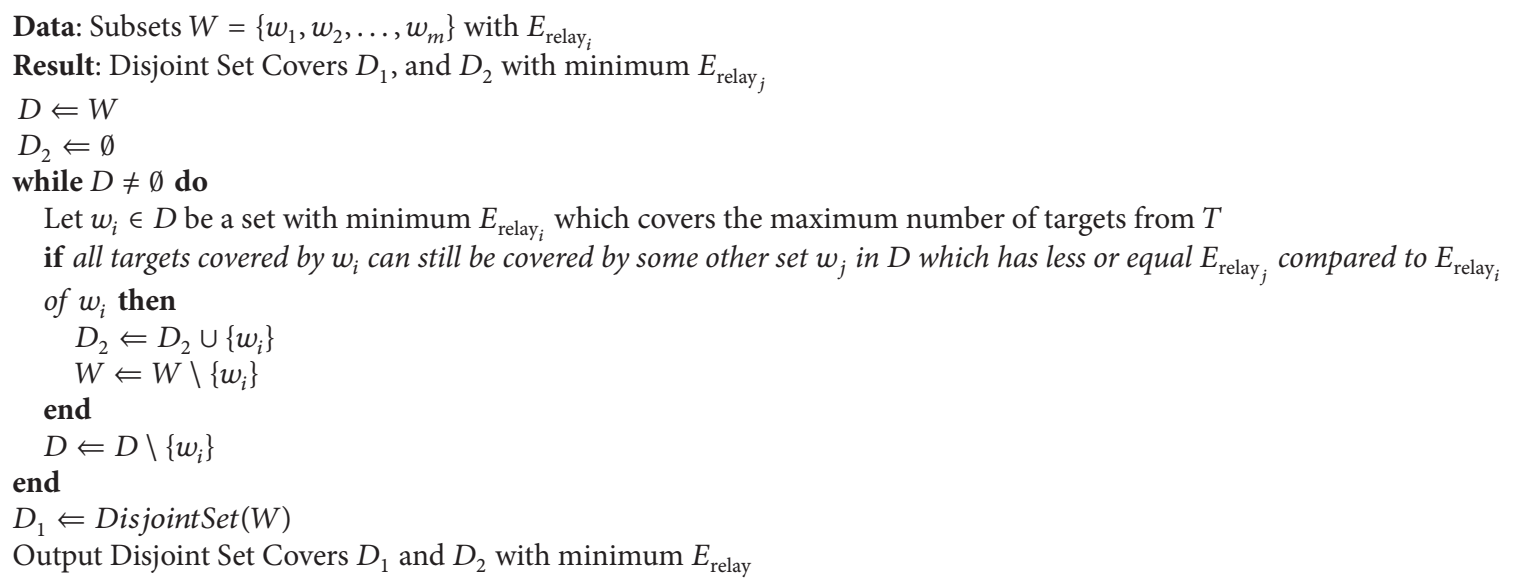

Algorithm 1: Energy Efficient Maximum Disjoint Coverage $\sqrt{m}$-approximation Algorithm (EMDC).

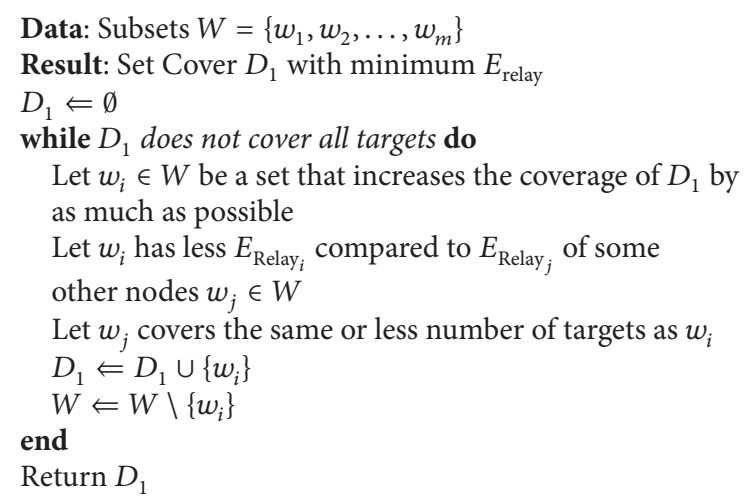

Algorithm 2: DisjointSet $(W)$ to compute set cover $D_{1}$ with minimum $E_{\text {relay }}$.

\section{Approximation Analysis}

Theorem 1. Approximation ratio of EMDC is not worse than $\sqrt{m}$, where $m$ denotes the number of elements in the set $T$.

Proof. Given a finite set of targets $T$ with $m$ elements, let us have a collection of subsets in $S$ which cover all $m$ elements of $T$. Let EMDC selects $w_{i}$ which has minimum $E_{\text {relay }_{i}}$ and adds it in set $D_{2}$ which can cover $k_{i}$ elements from the target set $T$. Let us say EMDC adds $M$ number of sets with minimum $E_{\text {relay }}$ to set $D_{2}$. Let us say in each iteration, $i$, number of targets covered by particular set $s_{i}$ with minimum $E_{\text {relay }}$ is represented by $m_{i}$. Then, the total number of targets covered by EMDC using $D_{2}$ with minimum $E_{\text {relay }}$ in $C$ iterations is given as follows:

$$
\left|D_{2}\right|=c_{1}+c_{2}+c_{3}+\cdots+c_{C} .
$$

Let us say in order to cover $T$ targets with $D_{2}$ with

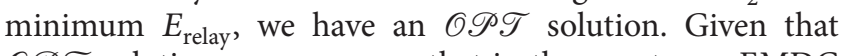
$\mathscr{O} \mathscr{P} \mathscr{T}$ solution, we can prove that in the worst case EMDC is a $\sqrt{m}$ approximation compared to $\mathscr{O} \mathscr{P} \mathscr{T}$. Let us say that EMDC computes $\left|D_{2}\right|$ which covers the number of targets which are greater than or equal to $\sqrt{m}$. In this case optimal algorithm $\mathscr{O} \mathscr{P T}$ is able to cover maximum $m$ elements. We can compare the maximum number of targets covered by both EMDC and $\mathscr{O} \mathscr{P T}$ as follows:

$$
\begin{gathered}
\left|D_{2}\right| \geq \sqrt{m} \\
|\mathcal{O P} \mathscr{T}| \leq m \Longrightarrow \\
\frac{|\mathcal{O P} \mathscr{T}|}{\left|D_{2}\right|} \leq \sqrt{m} \Longrightarrow \\
\sqrt{m} \text {-Approximation. }
\end{gathered}
$$

On the contrary, let the number of targets covered by $D_{2}$ using EMDC be less than or equal to $\sqrt{m}$. Let for each iteration, $i$, EMDC selects a set $w_{i}$ for $D_{2}$ and $w_{i}$ is the last available set in $W$. Let us say $w_{i}$ covers $m_{i-1}$ targets in each iteration. In worst case for all iterations and for each set $s_{i}$, EMDC loses at most $m_{i}\left(m_{i-1}\right)$ elements, where $1<i<M$. It means EMDC loses at most $m_{1}\left(m_{1}-1\right)+m_{2}\left(m_{2}-1\right)+m_{3}\left(m_{3}-\right.$ $1)+\cdots+m_{A}\left(m_{A}-1\right)$ denoted by $\left|\operatorname{Loss}_{D_{2}}\right|$, that is,

$$
\left|\operatorname{Loss}_{D_{2}}\right| \leq \sum_{i=1}^{A} m_{i}\left(m_{i}-1\right) \text {. }
$$

On the other hand, $|\mathscr{O P T}|$ is able to cover all the targets either part of $\left|D_{2}\right|$ or $\mid$ Loss $_{D_{2}} \mid$. For all the iterations, $A$, the maximum number of targets covered by $|\mathcal{O P T}|$ can be represented as follows:

$$
|\mathcal{O P T}| \leq\left|\operatorname{Loss}_{D_{2}}\right|+\left|D_{2}\right| \leq \sum_{i=1}^{A} m_{i}\left(m_{i}-1\right)+\sum_{i=1}^{A} m_{i} .
$$


TABLE 2: Simulation setting for EMDC.

\begin{tabular}{lc}
\hline Parameter & Value \\
\hline Simulation area & $1000 \times 1000$ \\
Node sensing range & $100 \mathrm{~m}$ \\
Transmission range & $200 \mathrm{~m}$ \\
Number of sensors & $50-150$ \\
Number of targets & $10-80$ \\
\hline
\end{tabular}

TABLE 3: Energy consumption model for EMDC.

\begin{tabular}{lc}
\hline State & Energy \\
\hline Listening & $10.9 \mathrm{~mA}$ \\
Transmit & $11.6 \mathrm{~mA}$ \\
Receive & $7.0 \mathrm{~mA}$ \\
\hline
\end{tabular}

We compare the number of targets covered by EMDC to the maximum number of targets covered by $\mathscr{O} \mathscr{P} \mathscr{T}$ algorithm as follows:

$$
\begin{aligned}
& \left|D_{2}\right| \leq m_{1}+m_{2}+m_{3}+\cdots+m_{A} \leq \sqrt{m} \\
& |\mathcal{O} \mathscr{P} \mathscr{T}| \leq m_{1}^{2}+m_{2}^{2}+m_{3}^{2}+\cdots+m_{A}^{2} \\
& \quad \leq\left(m_{1}+m_{2}+m k_{3}+\cdots+m_{A}\right)^{2} \\
& \quad \leq\left(m_{1}+m_{2}+m_{3}+\cdots+m_{A}\right) \cdot \sqrt{m} \Longrightarrow \\
& \quad \frac{|\mathcal{O P P}|}{m_{1}+m_{2}+m_{3}+\cdots+m_{A}} \leq \sqrt{m} \Longrightarrow \\
& \sqrt{m} \text {-Approximation. }
\end{aligned}
$$

From the above analysis, it is proved that EMDC is a $\sqrt{m}$ approximation.

\section{Performance Evaluations}

In our simulation scenarios, we use a sensor network with static sensor and target nodes randomly deployed in a $1000 \mathrm{~m}$ $\times 1000 \mathrm{~m}$ area. We specify the number of sensors and targets in each simulation scenario. In the sensing region, deployed targets and sensors are fixed during whole simulation time. If not stated otherwise, all deployed sensor nodes use uniform sensing range of $100 \mathrm{~m}$. Sensing range of each node is assumed to be twice of its transmission range. All results are averaged over 10 runs. The simulation parameters for the evaluation of EMDC are summarized in Table 2.

For the average network lifetime, energy model of MICA2 is considered. Each sensor is assumed to has an initial energy of $200 \mathrm{~J}$. We assume that energy consumed by each sensor is $\mathrm{J} /$ minute. Each simulation run lasts for 100 minutes. Table 3 summarizes the energy model for our simulations.

In the first scenario, sensors are varied from 50 to 150 to cover 50 targets deployed in an area of $1000 \times 1000$. All sensor nodes have uniform sensing range of $100 \mathrm{~m}$. Figure 3 shows the average disjoint set covers generated under varying number of sensor nodes for EMDC and DSC-MDC [60]. We can see that disjoint set covers tend to increase with an

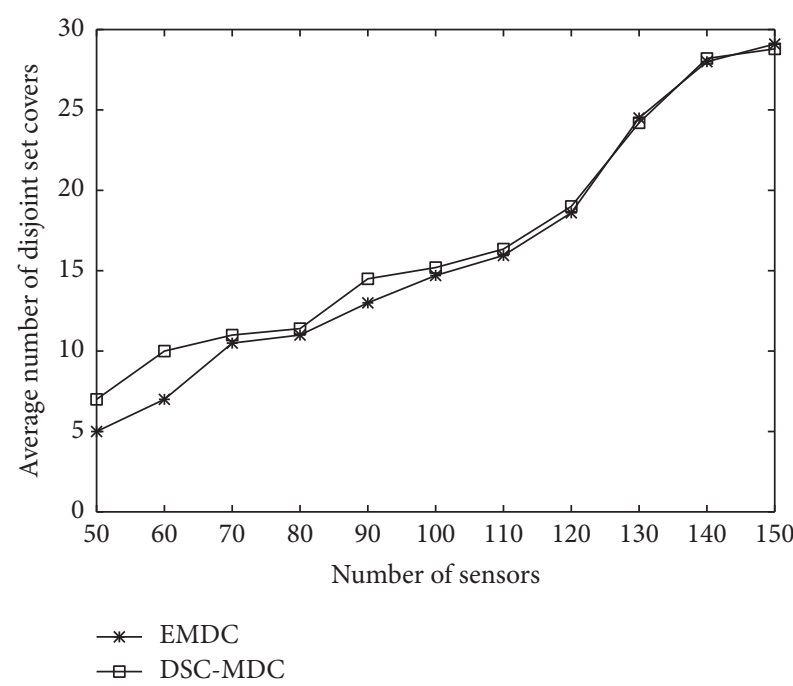

FIgURE 3: Number of disjoint set covers versus Number of Sensors.

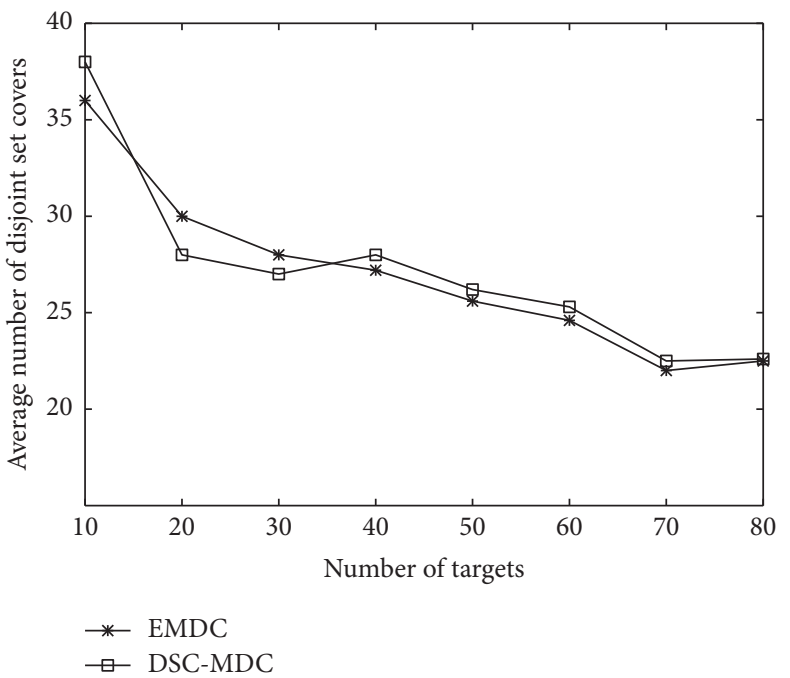

FIgURE 4: Number of disjoint set covers versus Number of Targets.

increase in sensor nodes for both EMDC and DSC-MDC. Disjoint set covers increase with an increase in the number of sensors, since more sensor nodes are available to qualify as set covers.

In the second scenario, 100 nodes with sensing range of $100 \mathrm{~m}$ are deployed in a fixed simulation area of $1000 \mathrm{~m} \times$ $1000 \mathrm{~m}$ to cover targets varying from 10 to 80 . Figure 4 shows disjoint set covers generated for varying number of targets. We observe that average disjoint set covers decreases as targets increase for both. The reason is that when targets increase while keeping the number of sensor nodes fixed, disjoint set covers decrease as likelihood of disjoint target coverage decreases. We can observe that EMDC has less disjoint set covers for all the targets due to the constraint of energy during the selection of set covers.

In third scenario, 100 sensors are deployed in a fixed simulation area of $1000 \mathrm{~m} \times 1000$ to cover 50 targets. Sensing range of each node is varied from $100 \mathrm{~m}$ to $500 \mathrm{~m}$. In Figure 5, 


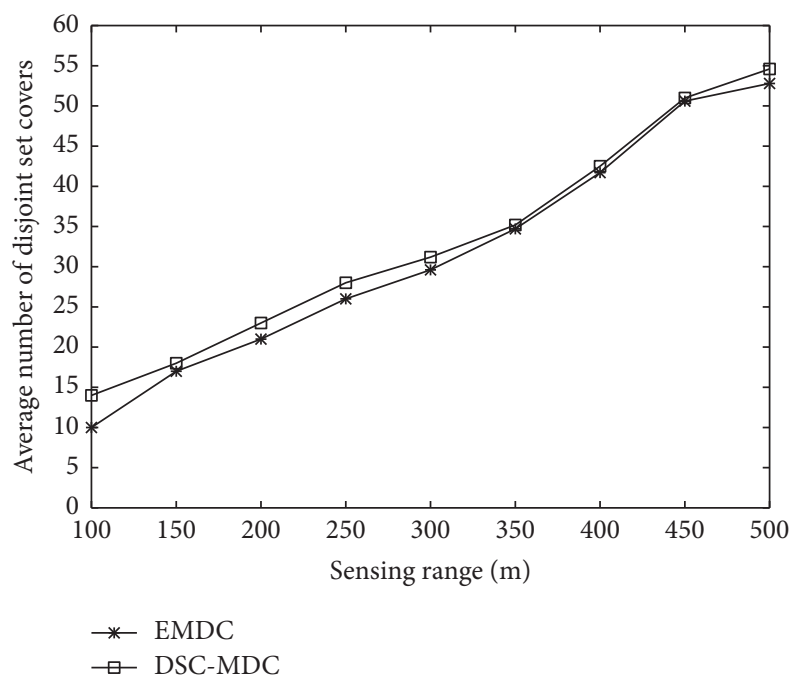

Figure 5: Number of disjoint set covers versus Sensing Range (m).

we observe the effect of sensing range on disjoint set covers. We can observe that for both the EMDC and DSC-MDC, with an increase in the sensing range, number of disjoint set covers increases. Disjoint set covers tend to increase consistently for all sensing ranges since each sensor node is able to cover more targets. This coverage increase results in an increase in disjoint set covers. However, we can observe that EMDC consistently has less disjoint set covers compared to the DSCMDC due to the minimum energy constraint on the selection of disjoint set covers.

Simulation parameters used in this experiment are same as used in scenario 1. All the nodes consume energy by using the energy model shown in Table 3. In Figure 6, we observe the effect of varying number of sensors on the average network lifetime. In case of DSC-MDC, more number of disjoint set covers is computed compared to EMDC since there is no constraint on the sensor nodes to qualify as a member of the set covers. Average network lifetime for both EMDC and DSC-MDC is directly proportional to disjoint sets produced. We already observed that EMDC has less disjoint set covers compared to the DSC-MDC as shown in Figure 4. However, we can observe that EMDC is still competitive to DSC-MDC for the average network lifetime because EMDC always selects disjoint set covers by taking into account the minimum energy.

Simulation parameters used in this experiment are the same as those used in scenario 2. All the nodes use the energy model as listed in Table 3. Figure 7 shows the network lifetime while increasing sensor nodes. It is clear from the Figure that network lifetime of EMDC is better than DSCMDC when number of nodes increases. In case of DSCMDC, assuming constant energy consumption for each node to forward the event, we can observe that average network lifetime consistently drops with an increase in targets. In order to monitor more targets, more energy is consumed to notify the event to the sink node. EMDC has better network lifetime compared to DSC-MDC because EMDC considers the nodes with minimum energy in order to qualify for the

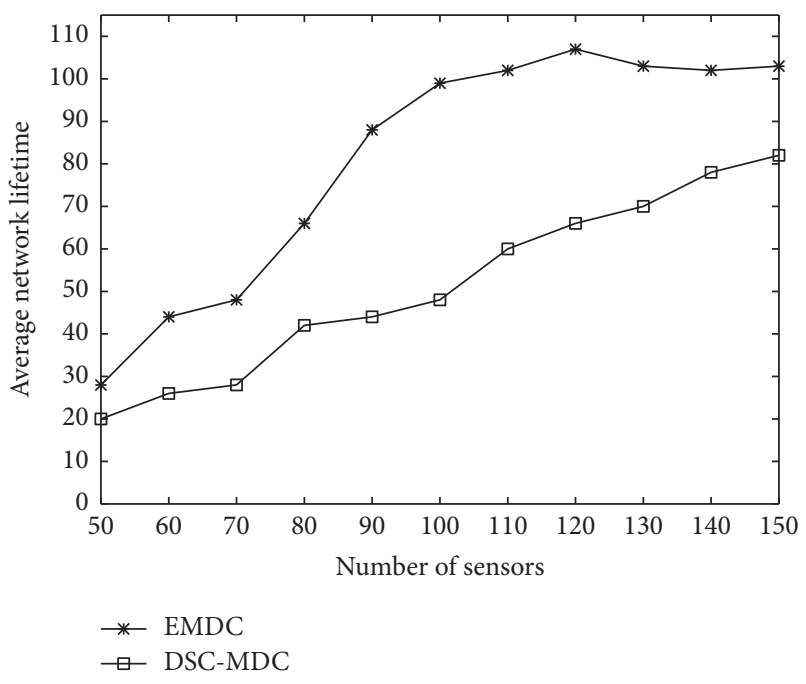

Figure 6: Network Lifetime versus Number of Sensors.

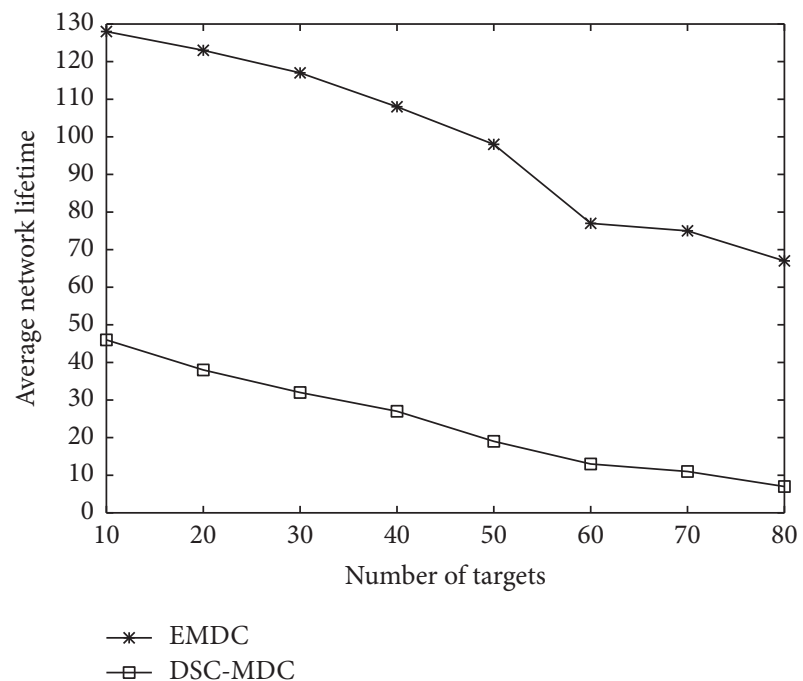

Figure 7: Network Lifetime versus Number of Targets.

set covers for both the disjoint sets. Results show that network lifetime is significantly improved when set covers are selected according to minimum energy. This observation is still valid when the number of targets tends to increase. For example, with 60 targets, average lifetime of EMDC is more than $80 \%$ better compared to DSC-MDC.

\section{Conclusion}

The proposed algorithm EMDC maximizes network lifetime by using two energy efficient disjoint set covers in a single hop WSN with a single sink. EMDC achieves energy efficiency and fault tolerance by selecting two disjoint set covers with minimum relay energy. On one hand, two disjoint set covers achieve fault tolerance by providing double coverage, and on the other hand they maximize network lifetime by considering the relay nodes with minimum energy required to report the event to the sink node. The experimental results reveal 
that EMDC achieves reasonable number of disjoint set covers to achieve fault tolerance under varying number of nodes and sensing ranges. Further, EMDC achieves better network lifetime compared to DSC-MDC for different number of nodes and sensing ranges.

\section{Conflicts of Interest}

The author declares no conflicts of interest.

\section{References}

[1] I. F. Akyildiz, W. Su, Y. Sankarasubramaniam, and E. Cayirci, "Wireless sensor networks: a survey," Computer Networks, vol. 38, no. 4, pp. 393-422, 2002.

[2] P. Guo, J. Wang, X. H. Geng, C. S. Kim, and J.-U. Kim, "A variable threshold-value authentication architecture for wireless mesh networks," Journal of Internet Technology, vol. 15, no. 6, pp. 929935, 2014.

[3] J. Shen, H. Tan, J. Wang, J. Wang, and S. Lee, "A novel routing protocol providing good transmission reliability in underwater sensor networks," Journal of Internet Technology, vol. 16, no. 1, pp. 171-178, 2015.

[4] Y. Liu, N. Xiong, Y. Zhao, A. V. Vasilakos, J. Gao, and Y. Jia, "Multi-layer clustering routing algorithm for wireless vehicular sensor networks," IET Communications, vol. 4, no. 7, pp. 810$816,2010$.

[5] H. Sajedi and Z. Saadati, "A hybrid structure for data aggregation in wireless sensor network," Journal of Computational Engineering, vol. 2014, Article ID 395868, 7 pages, 2014.

[6] P. Li, S. Guo, S. Yu, and A. V. Vasilakos, "Reliable multicast with pipelined network coding using opportunistic feeding and routing," IEEE Transactions on Parallel and Distributed Systems, vol. 25, no. 12, pp. 3264-3273, 2014.

[7] A. Sangwan and R. P. Singh, "Survey on coverage problems in wireless sensor networks," Wireless Personal Communications, vol. 80, no. 4, pp. 1475-1500, 2015.

[8] B. Wang, "Coverage problems in sensor networks: a survey," ACM Computing Surveys, vol. 43, no. 4, article no. 32, 2011.

[9] Y. Yao, Q. Cao, and A. V. Vasilakos, "EDAL: an energy-efficient, delay-aware, and lifetime-balancing data collection protocol for heterogeneous wireless sensor networks," IEEE/ACM Transactions on Networking, vol. 23, no. 3, pp. 810-823, 2015.

[10] M. Ashouri, Z. Zali, S. R. Mousavi, and M. R. Hashemi, "New optimal solution to disjoint set K-coverage for lifetime extension in wireless sensor networks," IET Wireless Sensor Systems, vol. 2, no. 1, pp. 31-39, 2012.

[11] Y. Lin, J. Zhang, H. S.-H. Chung, W. H. Ip, Y. Li, and Y.H. Shi, "An ant colony optimization approach for maximizing the lifetime of heterogeneous wireless sensor networks," IEEE Transactions on Systems, Man and Cybernetics Part C: Applications and Reviews, vol. 42, no. 3, pp. 408-420, 2012.

[12] K. Islam and S. G. Akl, "Target monitoring in wireless sensor networks: a localized approach," Ad-Hoc and Sensor Wireless Networks, vol. 9, no. 3-4, pp. 223-237, 2010.

[13] J. S. Wilson, Sensor Technology Handbook, Newnes, Oxford, UK, 2004.

[14] D. G. Costa and L. A. Guedes, "The coverage problem in videobased wireless sensor networks: a survey," Sensors, vol. 10, no. 9, pp. 8215-8247, 2010.
[15] X. Wang, G. Xing, Y. Zhang, C. Lu, R. Pless, and C. Gill, "Integrated coverage and connectivity configuration in wireless sensor networks," in Proceedings of the First International Conference on Embedded Networked Sensor Systems (SenSys '03), pp. 28-39, Los Angeles, Calif, USA, November 2003.

[16] D. Tian and N. D. Georganas, "A coverage-preserving node scheduling scheme for large wireless sensor networks," in Proceedings of the 1st ACM International Workshop on Wireless Sensor Networks and Applications, pp. 32-41, September 2002.

[17] J. Carle and D. Simplot-Ryl, "Energy-efficient area monitoring for sensor networks," Computer, vol. 37, no. 2, pp. 40-46, 2004.

[18] S. Slijepcevic and M. Potkonjak, "Power efficient organization of wireless sensor networks," in Proceedings of the International Conference of Communications (ICC '01), pp. 472-476, Helsinki, Finland, June 2001.

[19] K. Kar and S. Banerjee, "Node placement for connected coverage in sensor networks," in Proceedings of the Conference on Modeling and Optimization in Mobile, AdHoc and Wireless Networks, 2003.

[20] J. Luo and S. Zou, "Strong $k$-barrier coverage for one-way intruders detection in wireless sensor networks," International Journal of Distributed Sensor Networks, vol. 2016, Article ID 3807824, 16 pages, 2016.

[21] M. Cardei, D. MacCallum, M. X. Cheng et al., "Wireless sensor networks with energy efficient organization," Journal of Interconnection Networks, vol. 3, no. 4, pp. 213-229, 2002.

[22] J. Wu and H. Li, "On calculating connected dominating set for efficient routing in ad hoc wireless networks," in Proceedings of the ACM Workshop on Discrete Algorithms and Methods for Mobile Computing and Communications, pp. 7-14, August 1999.

[23] M. Cardei and D.-Z. Du, "Improving wireless sensor network lifetime through power aware organization," Wireless Networks, vol. 11, no. 3, pp. 333-340, 2005.

[24] F. Ye, G. Zhong, S. Lu, and L. Zhang, "Energy efficient robust sensing coverage in large sensor networks," Tech. Rep., UCLA, 2002.

[25] H. Zhang and J. C. Hou, "Maintaining sensing coverage and connectivity in large sensor networks," Tech. Rep. UIUCDCSR-2003-2351, Department of Computer Science, University of Illinois at Urbana, Champaign, Ill, USA, 2003.

[26] J. Chen, J. Li, and T. H. Lai, "Energy-efficient intrusion detection with a barrier of probabilistic sensors: global and local," IEEE Transactions on Wireless Communications, vol. 12, no. 9, pp. 4742-4755, 2013.

[27] T.-W. Sung and C.-S. Yang, "Voronoi-based coverage improvement approach for wireless directional sensor networks," Journal of Network and Computer Applications, vol. 39, no. 1, pp. 202-213, 2014.

[28] M. R. Senouci, A. Mellouk, and K. Assnoune, "Localized movement-assisted sensordeployment algorithm for holedetection and healing," IEEE Transactions on Parallel and Distributed Systems, vol. 25, no. 5, pp. 1267-1277, 2014.

[29] E. Yildiz, K. Akkaya, E. Sisikoglu, and M. Y. Sir, "Optimal camera placement for providing angular coverage in wireless video sensor networks," IEEE Transanctions on Computing, vol. 63, no. 7, pp. 1812-1825, 2014.

[30] L. Kong, M. Zhao, X.-Y. Liu et al., "Surface coverage in sensor networks," IEEE Transactions on Parallel and Distributed Systems, vol. 25, no. 1, pp. 234-243, 2014.

[31] Y.-G. Fu, J. Zhou, and L. Deng, "Surveillance of a 2D plane area with 3D deployed cameras," Sensors (Switzerland), vol. 14, no. 2, pp. 1988-2011, 2014. 
[32] C. Qiu and H. Shen, "A delaunay-based coordinate-free mechanism for full coverage in wireless sensor networks," IEEE Transanctions on Parallel Distributed System, vol. 25, pp. 500509, 2012.

[33] Y. Wang and G. Cao, "Achieving full-view coverage in camera sensor networks," ACM Transactions on Sensor Networks, vol. 10, no. 1, article 3, 2013.

[34] D. Zorbas and T. Razafindralambo, "Prolonging network lifetime under probabilistic target coverage in wireless mobile sensor networks," Computer Communications, vol. 36, no. 9, pp. 1039-1053, 2013.

[35] D. Tao, S. Tang, H. Zhang, X. Mao, and H. Ma, "Strong barrier coverage in directional sensor networks," Computer Communications, vol. 35, no. 8, pp. 895-905, 2012.

[36] Z. Wang, J. Liao, Q. Cao, H. Qi, and Z. Wang, "Achieving kbarrier coverage in hybrid directional sensor networks," IEEE Transactions on Mobile Computing, vol. 13, no. 7, pp. 1443-1455, 2014.

[37] T. H. Cormen, C. Stein, R. L. Rivest, and C. E. Leiserson, Introduction to Algorithms, McGraw-Hill Higher Education, 2001.

[38] S. S. Dhillon and K. Chakrabarty, "Sensor placement for effective coverage and surveillance in distributed sensor networks," in Proceedings of the IEEE Wireless Communications and Networking Conference: The Dawn of Pervasive Communication (WCNC '03), vol. 3, pp. 1609-1614, March 2003.

[39] S. S. Dhillon, K. Chakrabarty, and S. S. Iyengar, "Sensor placement for grid coverage under imprecise detections," in Proceedings of the 5th International Conference on Information Fusion (FUSION '02), pp. 1581-1587, July 2002.

[40] Y. Zou and K. Chakrabarty, "Uncertainty-aware and coverageoriented deployment for sensor networks," Journal of Parallel and Distributed Computing, vol. 64, no. 7, pp. 788-798, 2004.

[41] Z. Fang and J. Wang, "Sensor placement for grid coverage under imprecise detections," in Proceedings of the 3rd International Conference Wireless Algorithms, Systems, and Applications (WASA '08), pp. 188-199, Dallas, Tex, USA, 2008.

[42] F. Y. S. Lin and P. L. Chiu, "A near-optimal sensor placement algorithm to achieve complete coverage-discrimination in sensor networks," IEEE Communications Letters, vol. 9, no. 1, pp. 43-45, 2005.

[43] F. Y. S. Lin and P. L. Chiu, "Energy-efficient sensor network design subject to complete coverage and discrimination constraints," in Proceedings of the IEEE International Conference on Sensor and Ad hoc Communication and Networks, pp. 586-593, 2005.

[44] M. Cardei, M. T. Thai, Y. Li, and W. Wu, "Energy-efficient target coverage in wireless sensor networks," in Proceedings of the IEEE International Conference on Computer Communications (INFOCOM '05), pp. 1976-1984, March 2005.

[45] M. X. Cheng, L. Ruan, and W. Wu, "Coverage breach problems in bandwidth-constrained sensor networks," ACM Transactions on Sensor Networks, vol. 3, no. 2, article 12, 2007.

[46] Z. Abrams, A. Goel, and S. Plotkin, "Set k-cover algorithms for energy efficient monitoring in wireless sensor networks," in Proceedings of the Symposium on Information Processing in Sensor Networks, pp. 26-27, Berkeley, Calif, USA, April 2004.

[47] A. Deshpande, S. Khuller, A. Malekian, and M. Toossi, "Energy efficient monitoring in sensor networks," in Proceedings of the International Conference on Theoretical Informatics, pp. 7-11, 2008.
[48] I. Cardei and M. Cardei, "Energy-efficient connected-coverage in wireless sensor networks," International Journal of Sensor Networks, vol. 3, no. 3, pp. 201-210, 2008.

[49] P. Ostovari, M. Dehghan, and J. Wu, "Connected point coverage in wireless sensor networks using robust spanning trees," in Proceedings of the 31st International Conference on Distributed Computing Systems Workshops (ICDCSW '11), pp. 287-293, Minneapolis, Minn, USA, June 2011.

[50] M. Saravanan and M. Madheswaran, "A hybrid optimized weighted minimum spanning tree for the shortest intrapath selection in wireless sensor network," Mathematical Problems in Engineering, vol. 2014, Article ID 713427, 8 pages, 2014.

[51] R. Lachowski, M. E. Pellenz, M. C. Penna, E. Jamhour, and R. D. Souza, "An efficient distributed algorithm for constructing spanning trees in wireless sensor networks," Sensors (Switzerland), vol. 15, no. 1, pp. 1518-1536, 2015.

[52] Q. Zhao and M. Gurusamy, "Lifetime maximization for connected target coverage in wireless sensor networks," IEEE/ACM Transactions on Networking, vol. 16, no. 6, pp. 1378-1391, 2008.

[53] D. Zorbas, D. Glynos, P. Kotzanikolaou, and C. Douligeris, "Solving coverage problems in wireless sensor networks using cover sets," Ad Hoc Networks, vol. 8, no. 4, pp. 400-415, 2010.

[54] J. Yu, X. Deng, D. Yu, G. Wang, and X. Gu, "CWSC: connected kcoverage working sets construction algorithm in wireless sensor networks," International Journal of Electronics and Communications, vol. 67, no. 11, pp. 937-946, 2013.

[55] K.-P. Shih, D.-J. Deng, R.-S. Chang, and H.-C. Chen, "On connected target coverage for wireless heterogeneous sensor networks with multiple sensing units," Sensors, vol. 9, no. 7, pp. 5173-5200, 2009.

[56] J. Yu, Y. Chen, L. Ma, B. Huang, and X. Cheng, "On connected target k-coverage in heterogeneous wireless sensor networks," Sensors, vol. 16, no. 1, article 104, pp. 262-265, 2016.

[57] S. Yang, F. Dai, M. Cardei, and J. Wu, "On multiple point coverage in wireless sensor networks," in Proceedings of the 2nd IEEE International Conference on Mobile Ad-hoc and Sensor Systems (MASS '05), pp. 757-764, November 2005.

[58] I. Cardei, "Energy-efficient target coverage in heterogeneous wireless sensor networks," in Proceedings of the IEEE International Conference on Mobile Ad Hoc and Sensor Sysetems (MASS '06), pp. 397-406, Vancouver, Canada, October 2006.

[59] M. A. Khan, H. Hasbullah, and B. Nazir, "Multi-node repositioning technique for mobile sensor network," AASRI Procedia, vol. 5, pp. 85-91, 2013.

[60] S. Henna and T. Erlebach, "Approximating maximum disjoint coverage in wireless sensor networks," in Proceedings of the 12th International Conference on Ad-Hoc, Mobile, and Wireless Networks (ADHOC-NOW '13), Lecture Notes in Computer Science, pp. 148-159, Springer, July 2013. 


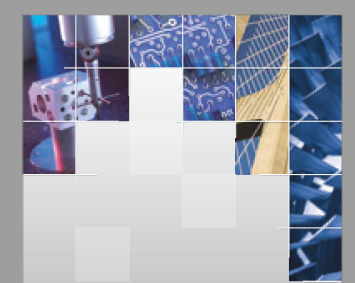

\section{Enfincering}
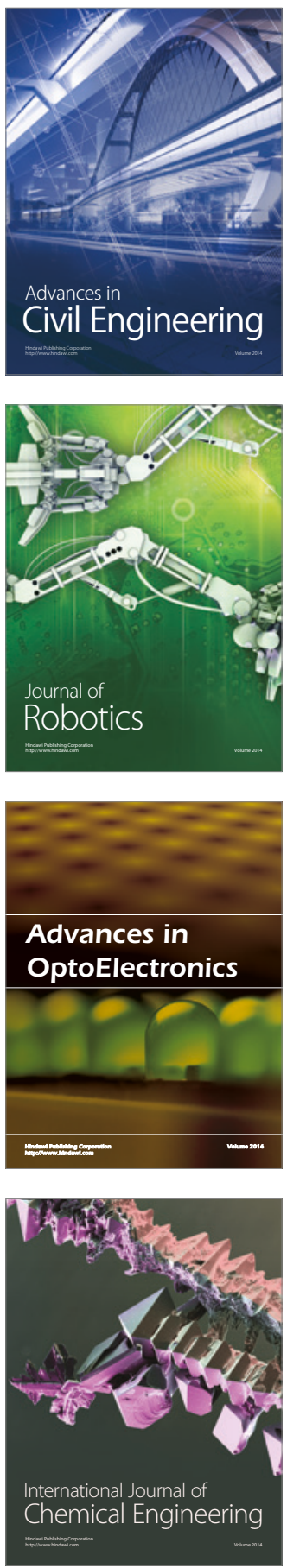

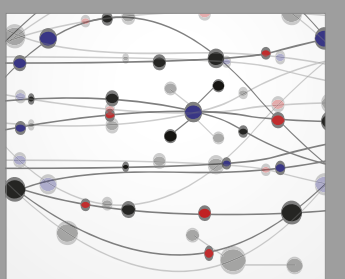

The Scientific World Journal

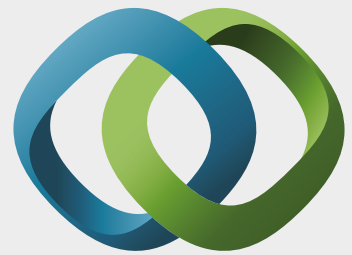

\section{Hindawi}

Submit your manuscripts at

https://www.hindawi.com
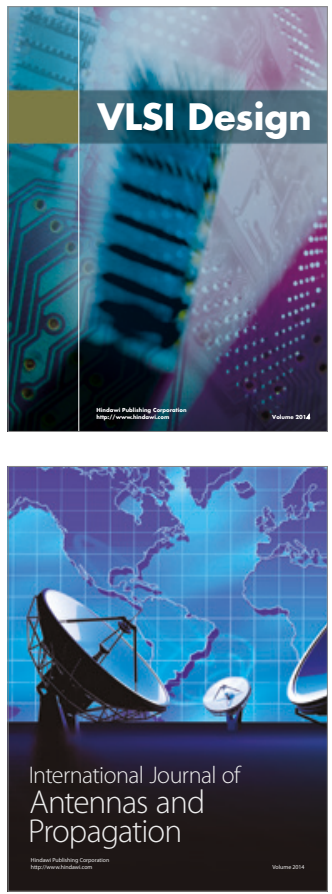

\section{Rotating}

Machinery
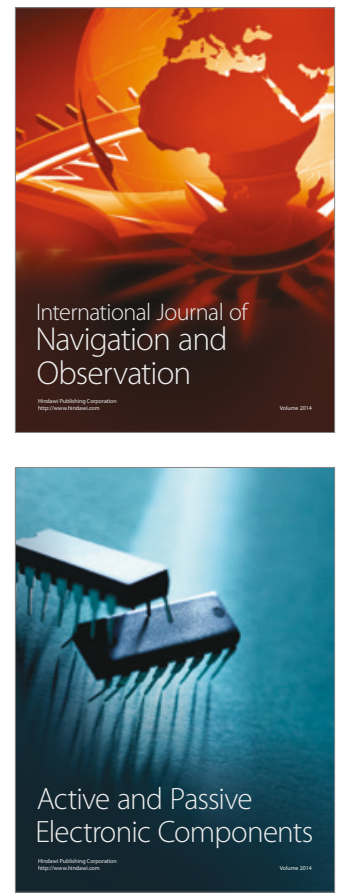
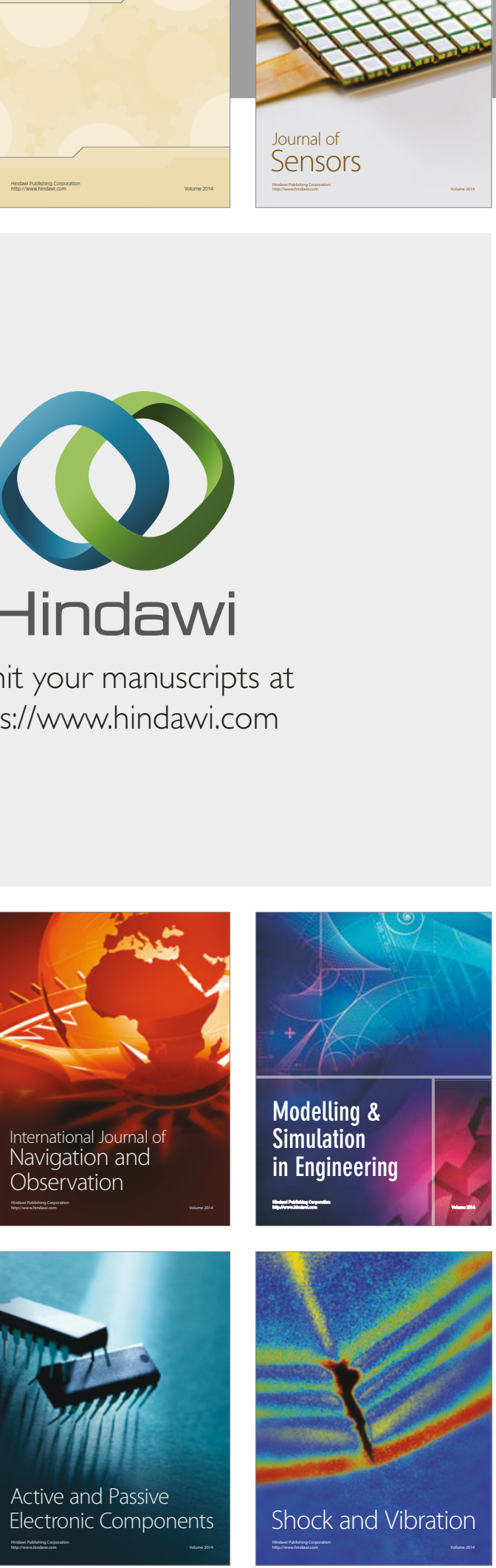
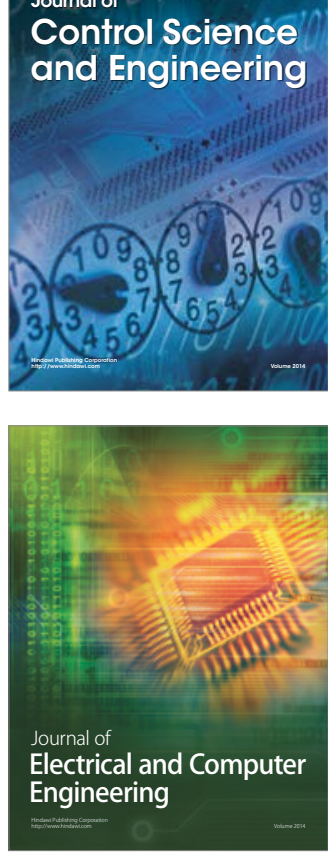

Distributed

Journal of

Control Science

and Engineering
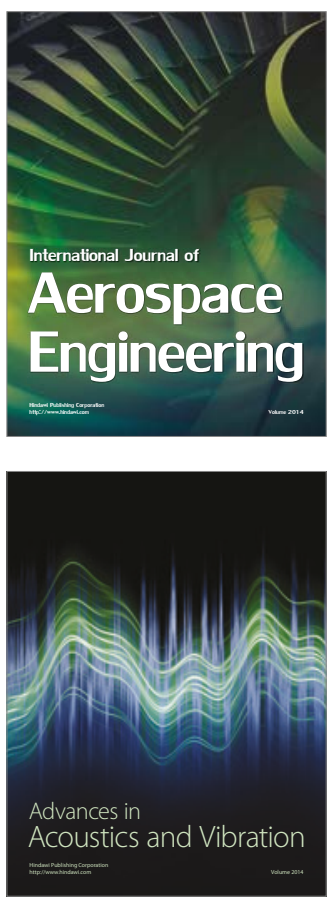

Sensor Networks 